\title{
Nanosecond pulsed field emission from single-gate metallic field emitter arrays fabricated by molding ${ }^{\text {a) }}$
}

\author{
S. Tsujino, ${ }^{\text {b) }}$ M. Paraliev, E. Kirk, T. Vogel, F. Le Pimpec, C. Gough, \\ S. Ivkovic, and H.-H. Braun \\ Paul Scherrer Institut, CH-5232 Villigen-PSI, Switzerland
}

(Received 19 October 2010; accepted 4 March 2011; published 23 March 2011)

\begin{abstract}
Electrically gated pulsed field emission from molybdenum field emitter arrays was studied. Single-gate field emitter array devices supported by metallic substrates were fabricated by a combination of molding and a self-aligned gate process. Devices were tested in a low-inductance cathode holder compatible with the high-acceleration electric field of a pulsed diode gun. Pulsed field emission down to $1.1 \mathrm{~ns}$ was observed for single-gate devices with $1.2 \times 10^{3}-1.2 \times 10^{5}$ emitter tips with $5 \mu \mathrm{m}$ array pitches. Integrating the field emitter arrays in a high-voltage pulsed diode gun, the authors demonstrated nanosecond field emission at an acceleration field of $30 \mathrm{MV} / \mathrm{m}$ at the cathode surface and acceleration of the field emission electron beam up to $300 \mathrm{keV}$. In addition, transverse beam emittance of the single-gate devices was measured with two different array sizes. (C) 2011 American Vacuum Society. [DOI: 10.1116/1.3569820]
\end{abstract}

\section{INTRODUCTION}

In this work, we explore the nanosecond pulsed field emission of an all-metallic field emitter array (FEA) cathode and its operation in a high-acceleration electric field. Accelerator applications which demand high brightness, such as compact X-ray free electron lasers, ${ }^{1-3}$ require compatibility with an acceleration field in the order of $100 \mathrm{MV} / \mathrm{m}$ to prevent emittance degradation of the electron beam. Operating high-acceleration-gradient devices in pulsed mode minimizes or eliminates breakdown, ${ }^{4,5}$ and thus switching of FEA field emission current in the nanosecond range or below is highly desirable. We are exploring fast electrical gating methods by testing FEAs in the pulsed diode electron gun test facility of the SwissFEL project at Paul Scherrer Institut. ${ }^{5,6}$

Compatibility of FEA with high-acceleration field is also important for terahertz vacuum electronic applications. ${ }^{7-9}$ Due to the Child-Langmuir space-charge limit, these applications require high-acceleration fields in order to generate proposed high-density beam currents in the order of $\sim 10^{3} \mathrm{~A} / \mathrm{cm}^{2} .{ }^{10-13}$ Laser pulses irradiating metallic and semiconductor cathodes ${ }^{14-17}$ or FEAs ${ }^{18,19}$ are commonly used to generate ultrafast electron bunches. However, fast electron bunches generated by using electrical gate pulses could have beneficial applications for vacuum electronic devices used from radio-frequency $(\mathrm{rf})^{20-25}$ to terahertz frequencies $^{7-9}$ and flash $\mathrm{x}$-ray tubes. ${ }^{26,27}$

\section{SAMPLE AND EXPERIMENTAL METHOD}

All-metallic FEAs were fabricated by molding and a selfaligned gate process. ${ }^{28-30}$ The emitter array consisted of pyramidal molybdenum tips with $\sim 1.5 \mu \mathrm{m}$ bases and $\sim 1.2 \mu \mathrm{m}$ heights aligned with $5 \mu \mathrm{m}$ pitch. The radii of

\footnotetext{
${ }^{a)}$ This article was presented at the 23rd International Vacuum Nanoelectronics Conference, Palo Alto, CA, 25-31 August 2010.

${ }^{b}$ Electronic mail: soichiro.tsujino@psi.ch
}

curvature of the emitter tips characterized by high-resolution scanning electron microscope were $\sim 5 \mathrm{~nm}$. These measurements were obtained by controlling the pit shape of the silicon mold substrate using the repeated oxidation process. ${ }^{30}$ The gate electrode consisted of a $0.5-\mu \mathrm{m}$-thick molybdenum film separated from the emitter substrate by a $1.2-\mu \mathrm{m}$-thick $\mathrm{SiO}_{2}$ insulation layer. The root-mean-square (rms) surface roughness of the flat gate electrode surface between emitters was measured by atomic-force microscope and equaled 2.9 nm. Around apertures with collars [Fig. 1(b)], the molybdenum film was rougher due to the angle of deposition. ${ }^{30}$ The FEA was supported by a 0.4-mm-thick electroplated nickel substrate. In the experiments described below, we used circular arrays with $1.2 \times 10^{3}$ and $1.2 \times 10^{5}$ tips of 0.223 and $2.258 \mathrm{~mm}$ diameters, respectively. Each tip had a gate aperture with a diameter $a_{\mathrm{ext}}$ of 1.4 or $2.4 \mu \mathrm{m}$. For noncircular shaped gate apertures, the diameter of the inscribed circle of the gate aperture was used as $a_{\text {ext }}$. The sample parameters are summarized in Table I. Figure 2 shows the field emission characteristics for FEA-1 (open squares) measured with a 400-ns-long $V_{\text {ge }}$ pulse bias and FEA-2 (filled circles) measured with a AC $50 \mathrm{~Hz}$ bias. The maximum current of FEA-2 was $37 \mathrm{~mA}$.

The electron gun beamline [Fig. 3(a)] consisted of a diode electron gun, a two-cell rf cavity operating at $1.5 \mathrm{GHz}$, and a diagnostics beamline. The diode electron gun accelerated electron beams up to $500 \mathrm{keV}$. The field emission electron pulse was monitored by the wall-current monitor and by the scintillating screens Scr-1 or Scr-2, located 1280 and 2030 $\mathrm{mm}$ downstream from the cathode, respectively. The gun beamline was equipped for emittance measurement including thermal emittance of the electron beam using the solenoidscan technique. In the solenoid-scan technique, the emittance of the beam is evaluated by analyzing the beam image size on Scr-1 or Scr-2 as a function of the magnetic field of the solenoid-1 or $-2 .^{31,32}$ Further details of the combined dioderf-cavity gun and the beamline are described in Ref. 6 . 

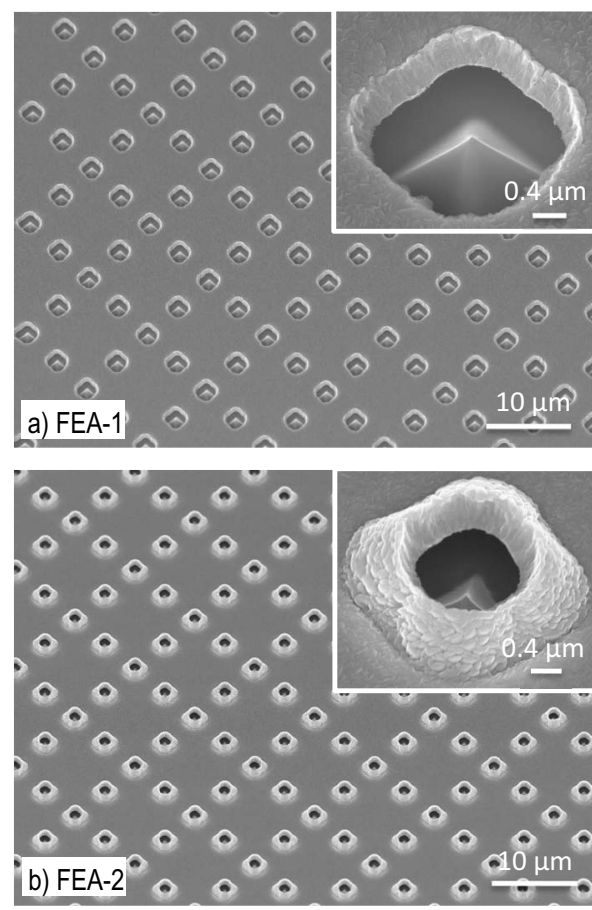

FIG. 1. Scanning electron microscope images of the single-gate FEAs with $1.2 \times 10^{5}$ emitter tips: (a) FEA-1 with the gate aperture diameter $a_{\text {ext }}$ equal to $2.4 \mu \mathrm{m}$; (b) FEA-2 with $a_{\mathrm{ext}}$ equal to $1.4 \mu \mathrm{m}$.

Figure 3(b) shows a schematic diagram of FEA chips integrated into the diode gun using the cathode holder. General construction of the cathode holder is depicted in Fig. 3(c). Emitter arrays consisting of $1.2 \times 10^{3}$ tips $(0.2 \mathrm{~mm}$ diameter $)$ or $1.2 \times 10^{5}$ tips ( $2.3 \mathrm{~mm}$ diameter) were placed at the center of $6.8 \mathrm{~mm}$ square chips. A circular gate electrode of $5.4 \mathrm{~mm}$ diameter overlapped the array for both sizes. The cathode holder had a $4 \mathrm{~mm}$ diameter opening with its inner edge in contact with the gate electrode. Figure 3(d) shows the electric field profile along the axis of the gun, where the anodecathode gap $g$, defined by the separation between $z_{\mathrm{B}}$ and $z_{\mathrm{C}}$, was equal to $4 \mathrm{~mm}$. This electric field profile was obtained from an electrostatic simulation based on the finite-element method. $F_{0}$ is defined as the value given by the cathode voltage divided by $g$. The left edge corresponds to the FEA surface, where the acceleration field on the FEA surface was approximately a factor of 2 smaller than $F_{0}$ due to the $1 \mathrm{~mm}$

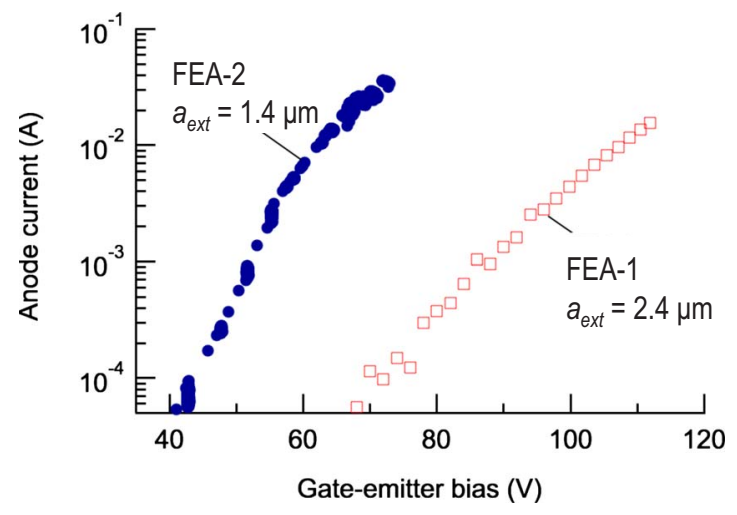

FIG. 2. (Color online) Relation between gate-emitter bias $V_{\text {ge }}$ and the anode current $I_{\mathrm{a}}$ for the two $1.2 \times 10^{5}$ tip FEAs, FEA-1 (open squares, $a_{\mathrm{ext}}$ equal to $2.4 \mu \mathrm{m}$ ) and FEA-2 (filled circles, $a_{\text {ext }}$ equal to $1.4 \mu \mathrm{m}$ ). DIODE: pulsed diode gun, RFC: rf-cavity, PS: pulsed solenoid, Sol-1 and Sol -2: solenoid, Scr-1 and Scr-2: scintillating screen monitor, K: cathode holder, and A: anode.

recess (the distance between $z_{\mathrm{A}}$ and $z_{\mathrm{B}}$ ). The exact reduction factor depended on $g$; for $g=4 \mathrm{~mm}$, the reduction factor was 0.52 as indicated in Fig. 3(d).

An air-core resonant transformer technology was used to generate the pulsed accelerating diode voltage. ${ }^{33}$ Figure 4 shows the accelerating voltage wave form of the diode gun, as measured by a calibrated capacitive divider. ${ }^{33}$ The full width half maximum (FWHM) duration of the first negative pulse peak maximum was $\sim 250$ ns. The accelerating voltage amplitude and the gap $g$ were variable. Thus, electron bunches could be accelerated up to $500 \mathrm{keV}$ in different acceleration conditions. ${ }^{34}$ In order to reduce breakdown probability, a critically coupled damped resonant system was used to provide an asymmetric oscillatory pulse [Fig. 4(a)]. To synchronize field emission with the first negative maximum of the accelerating voltage, time indicated by a dashed line in Fig. 4(b), we developed nanosecond pulser described below. The maximum repetition rate of the pulsed diode gun was $10 \mathrm{~Hz}$, being limited by the power capability of the main charging supply. Using mirror polished stainless steel coated by diamondlike carbon as cathode and anode, an acceleration field over $200 \mathrm{MV} / \mathrm{m}$ can be generated with $g$ of a few millimeters. ${ }^{5,6}$ The Child-Langmuir current density under such acceleration conditions is well above $\sim 4 \mathrm{kA} \mathrm{cm}^{-2}$. However, to evaluate the maximum current density more ac-

TABLE I. Parameters of FEAs used in this study. The normalized rms emittance $\varepsilon^{(n)}$ of two FEAs, FEA-3 and FEA-5, were measured by the solenoid-scan technique in horizontal and vertical directions.

\begin{tabular}{|c|c|c|c|c|c|}
\hline FEA & 1 & 2 & 3 & 4 & 5 \\
\hline Array diameter (mm) & 2.258 & 2.258 & 2.258 & 2.258 & 0.226 \\
\hline Number of the emitters & $1.2 \times 10^{5}$ & $1.2 \times 10^{5}$ & $1.2 \times 10^{5}$ & $1.2 \times 10^{5}$ & $1.2 \times 10^{3}$ \\
\hline Array pitch $(\mu \mathrm{m})$ & 5 & 5 & 5 & 5 & 5 \\
\hline Gate aperture diameter ${ }^{\mathrm{a}}(\mu \mathrm{m})$ & 2.4 & 1.4 & 2.4 & 2.4 & 1.4 \\
\hline$\varepsilon_{\text {horizontal }}^{(n)}(\mathrm{mm} \mathrm{mrad})$ & $\ldots$ & $\ldots$ & $3.3 \pm 0.1$ & $\ldots$ & $0.42 \pm 0.01$ \\
\hline$\varepsilon_{\text {vertical }}^{(n)}(\mathrm{mm} \mathrm{mrad})$ & $\ldots$ & $\ldots$ & $4.3 \pm 0.2$ & $\ldots$ & $0.24 \pm 0.01$ \\
\hline
\end{tabular}

${ }^{a}$ For noncircular shaped gate apertures, the diameter of the inscribed circle of the aperture was taken. 


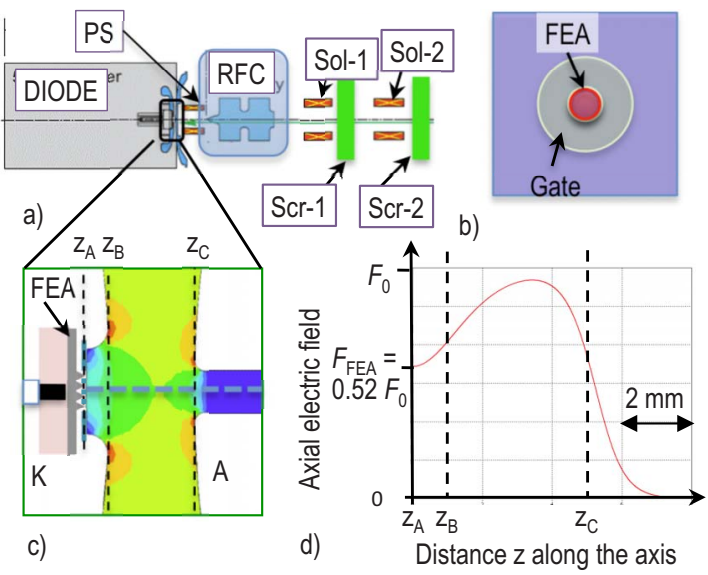

FIG. 3. (Color online) (a) Schematic diagram of the pulsed diode electron gun test facility of the SwissFEL project at the Paul Scherrer Institut, (b) schematic of the FEA device structure (top view), (c) closeup of the emitter cross-section enclosed in (a), and (d) the corresponding electric field profile along the gun axis. DIODE: diode gun, PS: pulsed solenoid, RFC: rf-cavity, Sol: solenoid, Scr: screen monitor.

curately, one should consider the two-dimensional geometry and the ratio between the gap and the emitter diameter, ${ }^{12,13}$ as well as the acceleration field profile along the gun axis.

The electron beam was accelerated and passed through an anode iris with a diameter of $2 \mathrm{~mm}$. When operating 1.2 $\times 10^{5}$ tip FEAs, a part of the field emission beam can be clipped by the anode iris since the array diameter is $0.6 \mathrm{~mm}$ larger than the anode iris diameter. However, electron loss is minimal because of lateral focusing of the electron beam
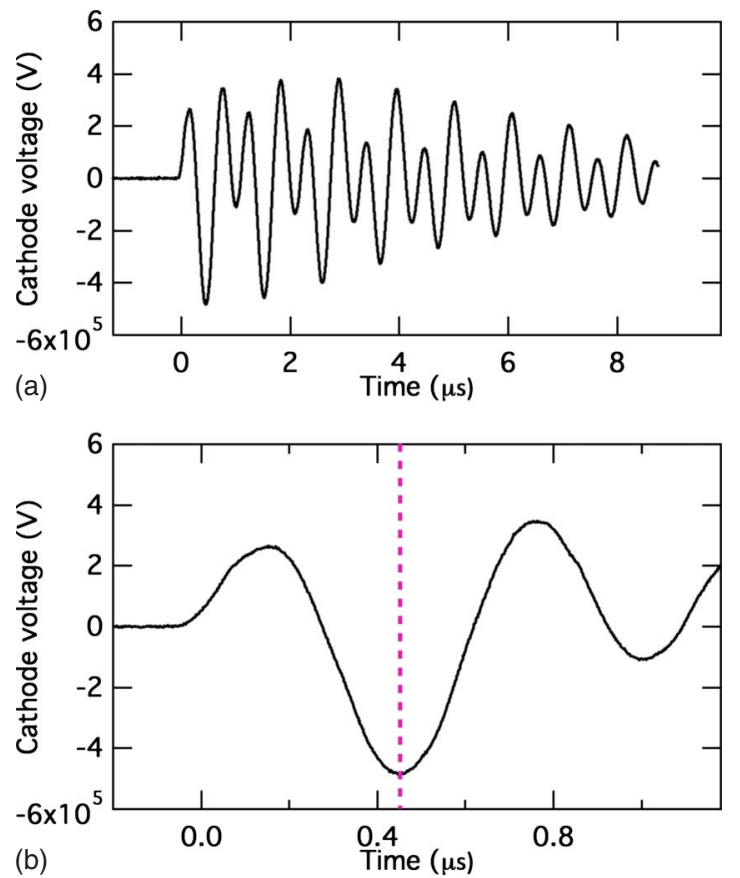

FIG. 4. (Color online) (a) Typical wave form of the oscillatory high-voltage pulse generated by the diode gun accelerator. (b) The first part of the oscillatory high-voltage pulse marked in (a). To accelerate electrons, the electron emission is triggered at the first negative peak of the pulse indicated by the vertical broken line.
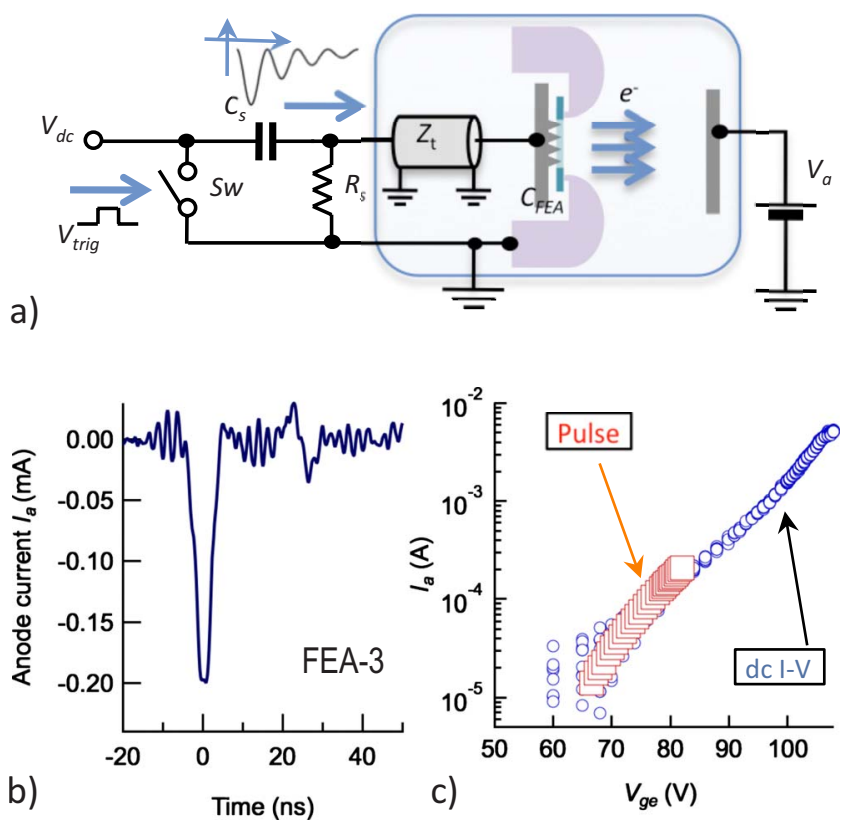

FIG. 5. (Color online) (a) Schematic diagram of the FEA driver. (b) 4-nswide pulsed field emission (FWHM) from FEA-3 $\left(1.2 \times 10^{5}\right.$ emitter array). (c) Comparison of pulsed $I-V$ and dc $I-V$.

near the FEA surface. This lateral focusing can be inferred from the correlation between increases in axial electric field and distance $z$ as shown in Fig. 3(d). ${ }^{35}$

We developed two fast pulsing schemes, which are depicted in Figs. 5(a) and 6(a). Although the FEA gate capacitance is $\sim 1 \mathrm{nF}$ due to the large area of the gate electrode, it is possible to drive the field emission down to nanosecond range by utilizing the low series resistance of the FEA chip (in the order of $10^{-4} \Omega$ ) and $R C$ time constant of the device,
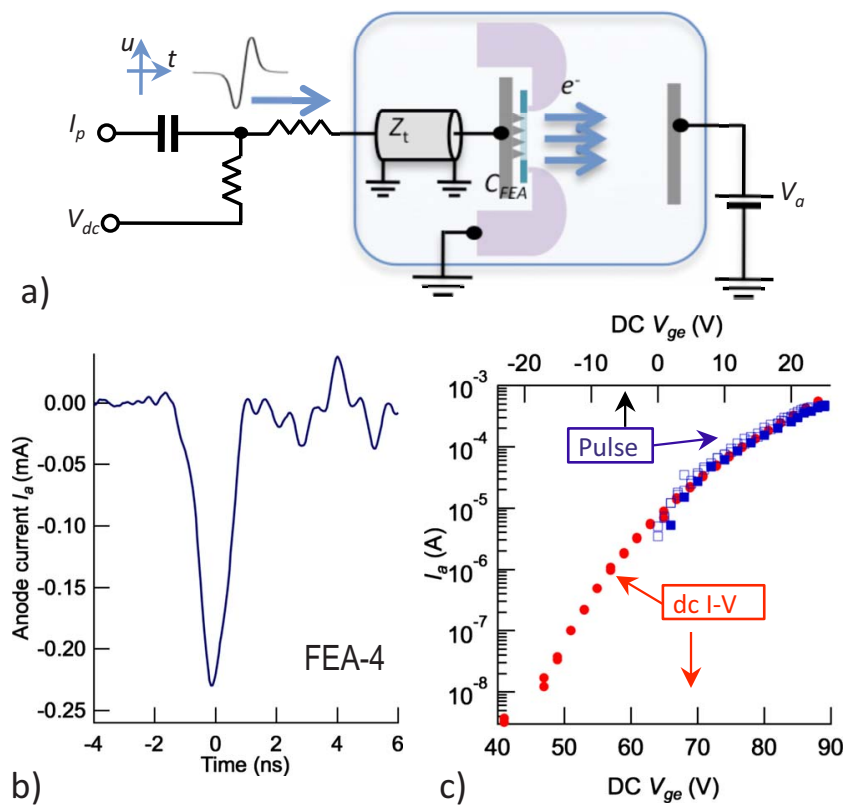

FIG. 6. (Color online) (a) Schematic diagram of the second FEA driver. (b) 1.1-ns-wide pulsed field emission from FEA-4 (1.2 $\times 10^{5}$ emitter array). (c) Comparison of pulsed $I-V$ and de $I-V$. 
which is several orders of magnitude less than $1 \mathrm{~ns}$. Therefore, nanosecond switching of FEAs is possible by devising appropriate driver circuits in combination with a lowinductance FEA holder [Fig. 3(c)]. In the first method, shown in Fig. 5(a), we excited a damped oscillatory gate pulse by switching a charged capacitor voltage. With this method, a sinusoidal-shaped gate pulse with 15 ns FWHM duration was applied to the FEA. When the trigger pulse $V_{\text {trig }}$ closed the transistor switch $\mathrm{Sw}$, the charge prestored in $C_{\mathrm{s}}$ by the driver supply voltage $V_{\mathrm{dc}}$ was partially transferred to the FEA via the low-impedance transmission line $Z_{\mathrm{t}}$ (characteristic impedance $11 \Omega$ and length of $100 \mathrm{~mm}$ ) within $\sim 15 \mathrm{~ns}$, applying a pulsed emitter bias $V_{\text {ge }}$ of $\sim 0.7 V_{\mathrm{dc}}$ to the FEA. In the second method, shown in Fig. 6(a), the FEA was biased by a current pulse of a few nanoseconds with negative polarity, immediately followed by a current pulse with the same amplitude and duration but with opposite polarity. Current pulses up to \pm 60 A with a duration of a few nanoseconds can be applied. A DC offset voltage $V_{\mathrm{dc}}$ is additionally available.

In the experiment, FEAs were first conditioned in a test chamber (not shown) with a maximum anode voltage of 4 $\mathrm{kV}$. The conditioning involved either repeatedly scanning the gate-emitter bias between 0 and a progressively higher value over a period of a few minutes or applying a $50 \mathrm{~Hz} \mathrm{AC}$ voltage with gradually increasing maximum amplitude. We continued this scan for tens of hours until current-voltage characteristics became stable. The emission current was collected by a stainless steel Faraday cup separated from the cathode by a distance of 40 or $10 \mathrm{~mm}$. The background pressure of the test chamber was in the order of $10^{-8}$ mbar. FEAs were further tested by a long pulse bias (400 ns or longer) or a short pulse bias (a few nanosecond) using the pulser shown in either Fig. 5(a) or 6(a). For the experiment in the high-voltage diode gun, FEAs were transferred through air after the conditioning and pulse test. The background pressure of the diode electron gun was also in the $10^{-8}$ mbar range.

In Sec. III, we first discuss the field emission experiments using the two pulsers depicted in Figs. 5(a) and 6(a). Then we discuss the pulsed field emission experiment in the diode electron gun using the first pulser [Fig. 5(a)]. Integration of the second pulser [Fig. 6(a)] into the diode electron gun, in combination with rf acceleration, is planned for a future experiment.

\section{RESULTS AND DISCUSSION}

First we discuss the results of pulsed field emission using two different pulsing techniques. Figure 5(b) shows the pulsed field emission from a $1.2 \times 10^{5}$ tip array (FEA-3) biased with the switched capacitor pulse driver depicted in Fig. 5(a). We observed an emission current pulse of $4.0 \mathrm{~ns}$ FWHM duration when the charging voltage of the driver was $117 \mathrm{~V}$. This generated a pulsed gate-emitter voltage $V_{\text {ge }}$ of $\sim 82 \mathrm{~V}$ with a duration of $15 \mathrm{~ns}$ for an anode-cathode distance of $40 \mathrm{~mm}$ and anode voltage $V_{\mathrm{a}}$ of $400 \mathrm{~V}$. The observed pulse duration was about four times shorter than the $V_{\text {ge }}$

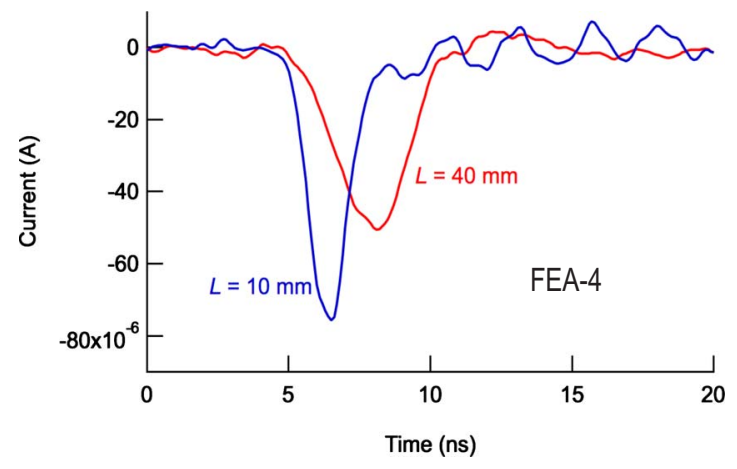

FIG. 7. (Color online) Pulsed field emission current wave form from FEA-4 $\left(1.2 \times 10^{5}\right.$ emitter tip array) driven by the pulser shown in Fig. 5(a) at two cathode-anode distances $L$ of 10 and $40 \mathrm{~mm}$ when the total $V_{\text {ge }}$ voltage was $\sim 70 \mathrm{~V}$.

pulse due to the nonlinear current-voltage characteristics of the field emission. A second current pulse was observed at $\sim 30$ ns after the main pulse with magnitude ten times smaller than the main pulse. This appears to be triggered by the second maximum of the damped oscillatory gate pulse. Figure 5(c) shows that the relation between anode current $I_{\mathrm{a}}$ and $V_{\text {ge }}$, with DC $V_{\text {ge }}$, compares well with the relation between pulsed $I_{\mathrm{a}}$ and $V_{\text {ge }}$ in the measured $V_{\text {ge }}$ range of $I_{\mathrm{a}}$ of up to $\sim 0.2 \mathrm{~mA}$.

Figure 6(b) shows the pulsed field emission from a 1.2 $\times 10^{5}$ tip array (FEA-4) using the pulser in Fig. 6(a). A pulsed $I_{\mathrm{a}}$ with a FWHM duration of $1.1 \mathrm{~ns}$ was observed for an anode-cathode distance of $10 \mathrm{~mm}$ and $V_{\mathrm{a}}$ of $800 \mathrm{~V}$. In this measurement, the estimated pulsed $V_{\text {ge }}$ value was $86 \mathrm{~V}$, corresponding to an injection current pulse amplitude of $\pm 56 \mathrm{~A}$. A DC-bias voltage $V_{\mathrm{dc}}$ of $-2 \mathrm{~V}$ was also applied at the same time. Figure 6(c) compares the dc $I_{\mathrm{a}}-V_{\text {ge }}$ relation with the pulsed measurement obtained by scanning $V_{\mathrm{dc}}$, with the pulsed $V_{\text {ge }}$ fixed at $\sim 64 \mathrm{~V}$. The two measurements agreed well for $I_{\mathrm{a}}$ up to $0.5 \mathrm{~mA}$. It was found that $V_{\mathrm{a}}$ and anodecathode separation $L$ impacted the observed current pulse duration over this time scale. As depicted in Fig. 7, when $L$ was increased from 10 to $40 \mathrm{~mm}$, the pulse was delayed by $1.6 \pm 0.1 \mathrm{~ns}$ and became broader by $1.4 \pm 0.1 \mathrm{~ns}$. The approximate agreement between these two values can be ascribed to the Shockley-Ramo induction of the current to the anode circuit by electron motion. ${ }^{36,37}$

Further reduction of the pulse duration is feasible by using faster bias pulses in combination with, for example, an increase in current pulse amplitude and optimization of device geometry, including reduction of device capacitance. ${ }^{25}$ The $R C$ diffusion/propagation time $T^{(R C)}$ of the gate pulse over the FEA is one limiting factor for the field emission current pulse duration of the experimental FEA chip. By defining $T^{(R C)}$ as the time required to reach $5 \%$ of the voltage uniformity over the area with radius $r$ of $2 \mathrm{~mm}$, we found, by solving the two-dimensional diffusion equation for the voltage and current pulses, that $T^{(R C)}$ is equal to 


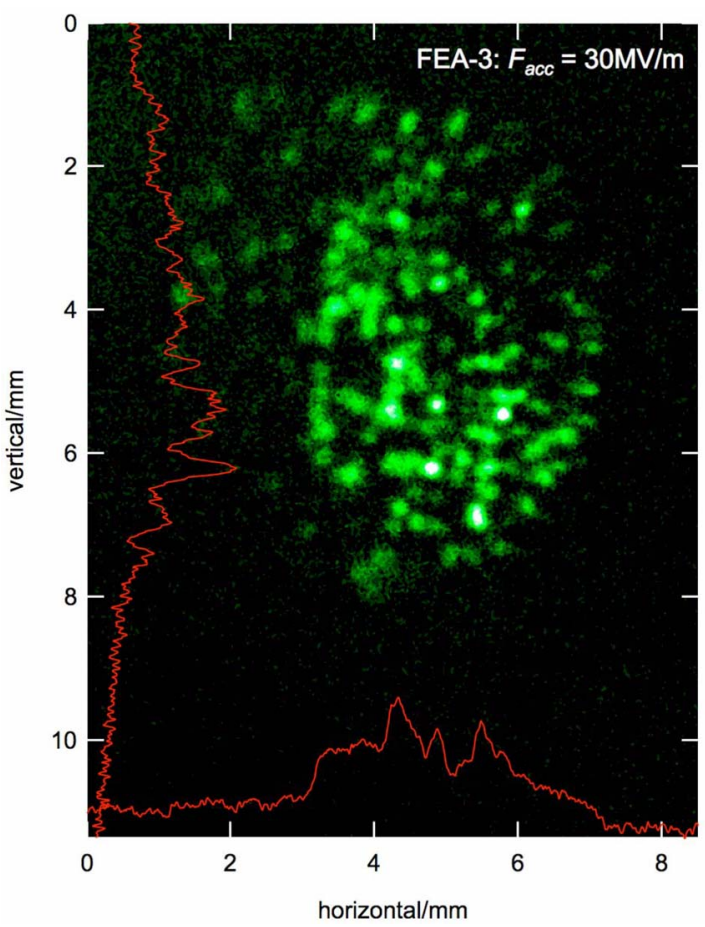

FIG. 8. (Color online) Pulsed field emission from a $2 \mathrm{~mm}$ diameter FEA, FEA-3 $\left(1.2 \times 10^{5}\right.$ tip array), under an acceleration field $F_{\text {acc }}$ of $30 \mathrm{MV} / \mathrm{m}$ on the FEA surface, imaged at screen Scr-1 of the pulsed diode electron gun.

$$
T^{(R C)} \cong 0.6 r^{2} / D^{(R C)},
$$

where $D^{(R C)}$ is the diffusion coefficient given by the following:

$$
D^{(R C)}=\frac{d h}{\rho \varepsilon \varepsilon_{0}} .
$$

In Eq. (2), $d$ is the thickness of the gate, $h$ is the thickness of $\mathrm{SiO}_{2}, \rho$ is the resistivity of the gate layer, $\varepsilon_{0}$ is the vacuum permittivity, and $\varepsilon$ is the dielectric constant of $\mathrm{SiO}_{2} . T^{(R C)}$ was estimated to be $32-150$ ps based on the measured values for the materials used in our FEAs ( $\rho$ of 20-100 $\mu \Omega \mathrm{cm}$ and $\varepsilon$ of 4.5$)$. $T^{(R C)}$ was 6-30 times shorter than the shortest pulse duration observed here.

Now we discuss the nanosecond operation of FEAs in the diode electron gun combined with the switched capacitor pulser depicted in Fig. 5(a). After transferring the pretested FEA to the gun through air and evacuating the gun to a base pressure of $\sim 10^{-8}$ mbar, field emission synchronized to the high-voltage pulse was achieved. The electron beam was then transported along the beamline to the screen Scr-1. Figure 8 shows the electron beam image of a $1.2 \times 10^{5}$ tip array (FEA-3) detected by screen Scr-1 when the acceleration field $F_{\text {acc }}$ on the FEA surface was $30 \mathrm{MV} / \mathrm{m}$. This $F_{\text {acc }}$ was generated by applying a high-voltage pulse of $-230 \mathrm{kV}$ to the cathode when the gap $g$ was $4 \mathrm{~mm}$. The pulsed solenoid current was $800 \mathrm{~A}$ and the focusing magnetic field of the double-solenoid sol-1 was $18.9 \mathrm{mT}$. In this optics setting, the field emission electron beam distribution from the FEA was imaged over $\mathrm{a} \sim 6 \mathrm{~mm}$ diameter area of the screen. The lines show the cross-sectional average intensity along the horizontal and vertical direction of the beam image. Under this high $F_{\text {acc }}$, we were able to extract a stable bunch charge of $\sim 1.5 \mathrm{pC}$ with $V_{\mathrm{ge}}$ equal to $\sim 75 \mathrm{~V}$. Using this FEA, the highest beam energy $300 \mathrm{keV}$ (at a $g$ of $15 \mathrm{~mm}$ and $F_{\text {acc }}$ of $11 \mathrm{MV} / \mathrm{m}$ ) was attained with a $0.1 \mathrm{pC}$ bunch charge and a $V_{\mathrm{ge}}$ of $\sim 50 \mathrm{~V}$. The highest bunch charge $(10 \mathrm{pC})$ was extracted with a $V_{\text {ge }}$ of $\sim 70 \mathrm{~V}$ under a $-230 \mathrm{kV}$ diode acceleration voltage ( $g$ of $15 \mathrm{~mm}$ and $F_{\text {acc }}$ of $8.5 \mathrm{MV} / \mathrm{m}$ ).

The observed granular emission image, shown in Fig. 8, indicates that not all of the emitter tips contributed to the emission. This can be ascribed to the nonuniform tip apex radii distributions as well as variations in cleanliness of tips. When the operation parameters were fixed in the conditions studied here, we found that the screen image and emission charge were stable for several hours during the tests. We also observed a flickering of some granule points without changing the total emission charge. It is likely that the tip apex nonuniformity may be partially compensated for by a tipblunting method which involves carefully increasing the pulse and dc-bias voltage and the anode voltage. ${ }^{38}$ However, this should perhaps be applied under low $F_{\text {acc }}$ and low $V_{\mathrm{a}}$ environment. During the FEA conditioning process in the separate test chamber with low $F_{\text {acc }}$, we observed an increase in emission current over a period of a few days. Both stepwise and smooth increases of the maximum current were observed. However, attempted in situ conditioning with nanosecond pulsed emission in the diode gun was so far unsuccessful because small arc events tend to lead to destruction of both the cathode and the cathode/anode fixtures.

Finally, we used the solenoid-scan technique to measure the transverse emittance of the field-emitted electron beam from FEAs with two different array sizes: FEA-3 $\left(1.2 \times 10^{5}\right.$ tip array with the array diameter of $2.258 \mathrm{~mm}$ ) and FEA-5 (a $1.2 \times 10^{3}$ tip array with the array diameter of $0.226 \mathrm{~mm}$ ). Figure 9(a) shows variations in the rms field emission electron beam diameters as a function of the magnetic field of the solenoid Sol-1, which is located $800 \mathrm{~mm}$ away from the anode [see Fig. 2(a)]. These were measured at the $F_{\text {acc }}$ of 9 $\mathrm{MV} / \mathrm{m}$ on the FEA surface with $-250 \mathrm{kV}$ diode voltage and $g$ of $15 \mathrm{~mm}$. The right panel of Fig. 9(a) shows the result for FEA-3 with a bunch charge of $\sim 0.5$ pC measured at Scr-1. The pulsed solenoid current was $800 \mathrm{~A}$. The left panel shows the result for FEA-5 measured at the second screen Scr-2, located $750 \mathrm{~mm}$ downstream from Scr-1. For the FEA-5 emittance measurement, the bunch charge was $\sim 0.2 \mathrm{pC}$ and the pulsed solenoid current was $775 \mathrm{~A}$. The beam diameters for each magnetic field setting were evaluated by fitting the electron beam intensity profile by a Gaussian distribution. The normalized rms emittance $\varepsilon^{(n)}$ of the beam was then evaluated by fitting the Twiss parameters to the measured magnetic field dependence of the beam diameter and optics of the beamline. ${ }^{6,31,32,39}$ The results of the fitting are indicated by the chain curves in Fig. 9(a). The $\varepsilon^{(n)}$ values are summarized in Table I. In Fig. 9(b), we show the values of $\varepsilon^{(n)}$ geometrically averaged in two directions. These results indicate that for both FEAs, $\varepsilon^{(n)}$ is proportional to the FEA 

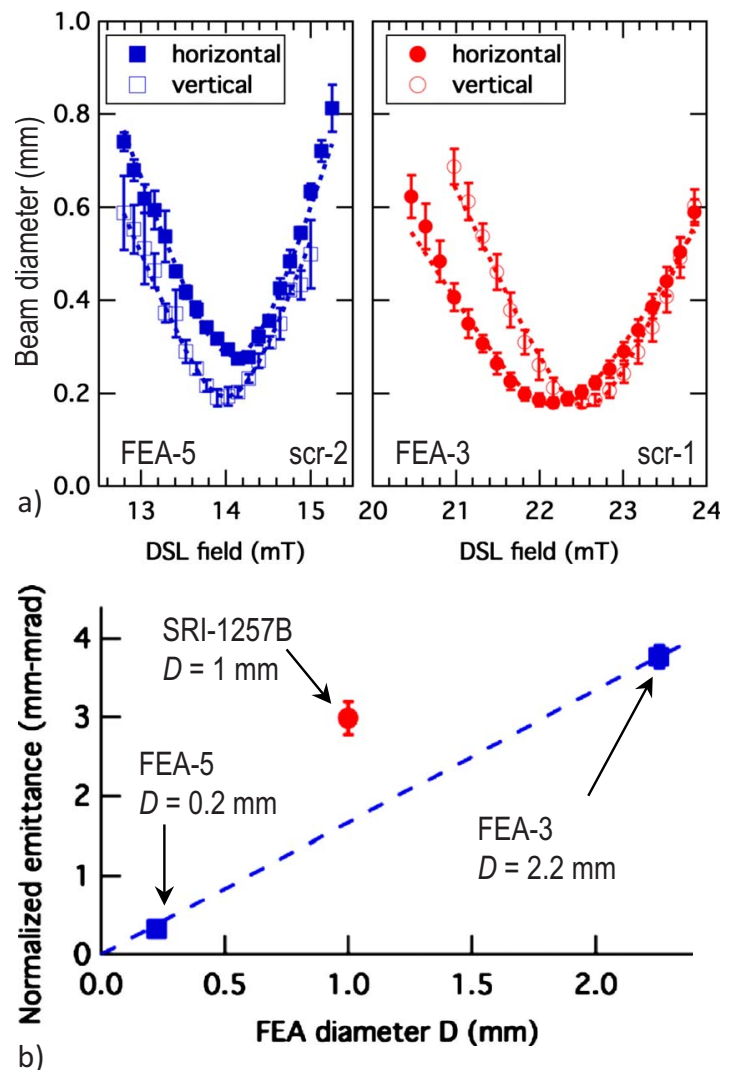

FIG. 9. (Color online) (a) Relation between the rms beam size and the sol-1 magnetic field (DSL field) for two FEAs with array diameters of $2.258 \mathrm{~mm}$ (FEA-3, $1.2 \times 10^{5}$ tip array), in right panel, and of $0.226 \mathrm{~mm}$ (FEA-5, 1.2 $\times 10^{3}$ tip array), in the left panel. (b) The relation between the FEA diameter $D$ and the $\varepsilon^{(n)}$ averaged over the two directions for the two FEAs and the previously reported $\varepsilon^{(n)}$ for a Spindt-FEA. The line shows the relation $\varepsilon^{(n)}$ $\approx 1.7 D$.

diameter $D$, described as $\varepsilon^{(n)} \approx 1.7 D$. In Fig. 9(b), we also show the previously reported $\varepsilon^{(n)}$ equal to $3.0 \pm 0.2 \mathrm{~mm} \mathrm{mrad}$ for a $5 \times 10^{4}$ tip and $1 \mathrm{~mm}$ diameter single-gate Spindt-FEA under $F_{\text {acc }}$ of $3.6 \mathrm{MV} / \mathrm{m}$, a $40 \mathrm{pC}$ bunch charge, and a $100 \mathrm{~ns}$ FWHM pulse width. ${ }^{31,32}$ This is $\sim 1.8$ times larger than the value obtained in the present measurement. We should note that the emittance value is highly sensitive to the parameters of the accelerator optics and the homogeneity of the emission beam. Therefore, further systematic study will be required to confirm the proportionality of the emittance value reported here and to clarify the impact of the FEA device structures and operation conditions, as well as the emission nonuniformity. ${ }^{40}$

\section{SUMMARY AND CONCLUSIONS}

In summary, we developed nanosecond pulsed field emission schemes for single-gate molybdenum FEA cathodes compatible with the diode electron gun of the SwissFEL test facility. Synchronization of nanosecond gated field emission from the FEA to the high-voltage pulse of the diode gun was realized. Compatibility of the FEA with an acceleration field of up to $30 \mathrm{MV} / \mathrm{m}$ was demonstrated. In addition, the solenoid-scan method was used to evaluate the normalized rms emittance of the FEA. Further reduction of the emission pulse width is foreseeable by optimization of the pulse scheme and the FEA device geometry. However, the emittance value urges implementation of a built-in focusing gate structure to realize a high-brightness cathode based on FEAs. ${ }^{2,41,42}$ Hence, the development of double-gate FEAs compatible with nanosecond gated emission will be the subject of research in the immediate future.

\section{ACKNOWLEDGMENTS}

The authors acknowledge A. F. Wrulich for his support and discussions on the FEA applications to SwissFEL, J. Gobrecht for his support and discussions on FEA fabrication, B. Beutner for discussion on the solenoid-scan emittance measurement method, and B. Haas and A. Lucke for their help in sample fabrication. This work was partially supported by the Swiss National Science Foundation Grant No. 200021-125084.

${ }^{1}$ M. Dehler, Proceedings of the 9th International Computational Accelerator Physics Conference (ICAP2006), Chamonix, France, 2-6 October 2006 (unpublished), pp. 114-117.

${ }^{2}$ C. M. Tang, A. C. Ting, and T. Swyden, Nucl. Instrum. Methods Phys. Res. A 318, 353 (1992).

${ }^{3}$ B. D. Patterson et al., New J. Phys. 12, 035012 (2010).

${ }^{4}$ J. Smedley, T. Srinivasan-Rao, T. Tsang, J. P. Ferrell, and K. Batchelor, Proceedings of the 17th International Conference on Application of Accelerators and Industry, AIP Conf. Proc. No. 680 (AIP, New York, 2003), p. 1054.

${ }^{5}$ M. Paraliev, C. Gough, S. Ivkovic, and F. le Pimpec, Proceedings in IEEE International Power Modulator Conference and High Voltage Conference, Atlanta, 2010 (unpublished), p. 3P30.

${ }^{6}$ R. Ganter et al., Phys. Rev. ST Accel. Beams 13, 093502 (2010).

${ }^{7}$ J. Urata, M. Goldstein, M. F. Kimmitt, A. Naumov, C. Platt, and J. E. Walsh, Phys. Rev. Lett. 80, 516 (1998).

${ }^{8}$ R. L. Ives, IEEE Trans. Plasma Sci. 32, 1277 (2004).

${ }^{9}$ J. H. Booske, Phys. Plasmas 15, 055502 (2008).

${ }^{10}$ C. D. Child, Phys. Rev. 32, 492 (1911).

${ }^{11}$ I. Langmuir, Phys. Rev. 2, 450 (1913).

${ }^{12}$ Y. Y. Lau, Phys. Rev. Lett. 87, 278301 (2001).

${ }^{13}$ J. W. Luginsland, Y. Y. Lau, R. J. Umstattd, and J. J. Watrous, Phys. Plasmas 9, 2371 (2002).

${ }^{14}$ J. A. Nation, L. Schächter, F. M. Mako, L. K. Len, W. Peter, C. M. Tang, and T. Srinivasan-Rao, Proc. IEEE 87, 865 (1999).

${ }^{15}$ R. Bossart, H. Braun, M. Dehler, and J. C. Godot, CERN Report No. CLIC, Note 297, 1996 (unpublished).

${ }^{16}$ K. Abrahamyan et al., Nucl. Instrum. Methods Phys. Res. A 528, 360 (2004).

${ }^{17}$ Y. Ding et al., Phys. Rev. Lett. 102, 254801 (2009).

${ }^{18}$ C. J. Chiang, D. R. Yankelevich, and J. P. Heritage, J. Appl. Phys. 106, 084505 (2009).

${ }^{19}$ S. Tsujino, P. Beaud, E. Kirk, T. Vogel, H. Sehr, J. Gobrecht, and A. Wrulich, Appl. Phys. Lett. 92, 193501 (2008).

${ }^{20}$ J. P. Calame, H. F. Gray, and J. L. Shaw, J. Appl. Phys. 73, 1485 (1993).

${ }^{21}$ H. Makishima, S. Miyano, H. Imura, J. Matsuoka, H. Takemura, and A. Okamoto, Appl. Surf. Sci. 146, 230 (1999).

${ }^{22}$ K. L. Jensen, Phys. Plasmas 6, 2241 (1999).

${ }^{23}$ D. R. Whaley, B. M. Gannon, C. R. Smith, C. M. Armstrong, and C. A. Spindt, IEEE Trans. Plasma Sci. 28, 727 (2000).

${ }^{24}$ D. R. Whaley, B. M. Gannon, V. O. Heinen, K. E. Kreischer, C. E. Holland, and C. A. Spindt, IEEE Trans. Plasma Sci. 30, 998 (2002).

${ }^{25}$ D. R. Whaley, R. Duggal, C. M. Armstrong, C. L. Bellew, C. E. Holland, and C. A. Spindt, IEEE Trans. Electron Devices 56, 896 (2009).

${ }^{26}$ R. Germer, J. Phys. E 12, 336 (1979).

${ }^{27}$ D. Rabinovich and B. Lourie, Acta Crystallogr., Sect. B: Struct. Sci. 43, 502 (1987).

${ }^{28}$ H. F. Gray and R. F. Greene, U.S. Patent No. 4,307,507 (29 December 
1981).

${ }^{29}$ M. Nakamoto, T. Haswegawa, T. Ono, T. Sakai, and N. Sakuma, Tech. Dig. - Int. Electron Devices Meet. 1996, 297.

${ }^{30}$ E. Kirk, S. Tsujino, T. Vogel, K. Jefimovs, J. Gobrecht, and A. Wrulich, J. Vac. Sci. Technol. B 27, 1813 (2009).

${ }^{31}$ S. C. Leemann, A. Streun, and A. F. Wrulich, Phys. Rev. ST Accel. Beams 10, 071302 (2007).

${ }^{32}$ S. C. Leemann, Ph.D. Thesis, Swiss Federal Institute of Technology Lausanne, 2007, pp. 184-195.

${ }^{33}$ M. Paraliev, C. Gough, and S. Ivkovic, Proceedings in IEEE International Power Modulator Conference and High Voltage Conference, Las Vegas, NV, 2008 (unpublished), p. 532.

${ }^{34}$ F. Le Pimpec, C. Gough, M. Paraliev, R. Ganter, C. Hauri, and S. Ivkovic, J. Vac. Sci. Technol. A 28, 1191 (2010).
${ }^{35}$ M. Reiser, Theory and Design of Charged Particle Beam Physics and Accelerator Technology (Weily-VCH Verlag GmbH \& Co. KGaA, Weinheim, 2008), p. 78.

${ }^{36}$ W. Shockley, J. Appl. Phys. 9, 635 (1938).

${ }^{37}$ S. Ramo, Proc. IRE 27, 584 (1939).

${ }^{38}$ P. R. Schwoebel, C. A. Spindt, and C. E. Holland, J. Vac. Sci. Technol. B 23, 691 (2005).

${ }^{39}$ F. Löhl, Ph.D. thesis, Universität Hamburg, 2005, pp. 9 and 10.

${ }^{40}$ K. L. Jensen, P. G. O'Shea, D. W. Feldman, and J. L. Shaw, J. Appl. Phys. 107, 014903 (2010).

${ }^{41}$ S. Tsujino, P. Helfenstein, E. Kirk, T. Vogel, C. Escher, and H. W. Fink, IEEE Electron Device Lett. 31, 1059 (2010).

${ }^{42}$ P. Helfenstein, E. Kirk, K. Jefimovs, T. Vogel, C. Escher, H. W. Fink, and S. Tsujino, Appl. Phys. Lett. 98, 061502 (2011). 